\title{
Effect of PEG-Polycation Chain Flexibility on siRNA Loaded Polyion Complex Micelles Assembly and Performance ${ }^{\dagger}$
}

\author{
Wenqian Yang ${ }^{1}$, Takuya Miyazaki ${ }^{2,3}$, Taehun Hong ${ }^{1}$ and Horacio Cabral 1,* \\ 1 Department of Bioengineering, Graduate School of Engineering, The University of Tokyo, 7-3-1 Hongou, \\ Tokyo 113-8656, Japan; yang-wenqian462@g.ecc.u-tokyo.ac.jp (W.Y.); \\ hongtaehun1125@g.ecc.u-tokyo.ac.jp (T.H.) \\ 2 Kanagawa Institute of Industrial Science and Technology, 705-1 Shimoimaizumi, Ebina, \\ Kanagawa 243-0435, Japan; takubsr@tmd.ac.jp \\ 3 Institute of Biomaterials and Bioengineering, Tokyo Medical and Dental University, \\ 2-3-10 Kanda-Surugadai, Chiyoda-ku, Tokyo 101-0062, Japan \\ * Correspondence: horacio@bmw.t.u-tokyo.ac.jp \\ + Presented at the 2nd International Online-Conference on Nanomaterials, 15-30 November 2020; \\ Available online: https://iocn2020.sciforum.net/.
}

Citation: Yang, W.; Miyazaki, T.; Hong, T.; Cabral, H. Effect of PEGPolycation Chain Flexibility on siRNA Loaded Polyion Complex Micelles Assembly and Performance. Mater Proc. 2021, 4, 88. https://doi. org/10.3390/IOCN2020-07985

Published: 12 November 2020

Publisher's Note: MDPI stays neutral with regard to jurisdictional claims in published maps and institutional affiliations.

Copyright: (c) 2020 by the authors. Licensee MDPI, Basel, Switzerland. This article is an open access article distributed under the terms and conditions of the Creative Commons Attribution (CC BY) license (http://creativecommons.org/licenses/by/4.0/).

\begin{abstract}
RNA interference (RNAi) has emerged as a promising therapeutic approach for the treatment of a wide range of disorders. Small interfering RNAs (siRNAs), i.e., non-coding doublestranded RNA molecules, have been mainly used for RNAi. Because siRNA is susceptible to enzymatic degradation and is rapidly cleared from the bloodstream, the success of RNAi is strongly related to the design of efficient delivery technologies. Among auspicious carriers for siRNA, polymeric micelles self-assembled by polyion complexation between block ionomers and siRNA have attracted much attention due to their well-defined size, efficient complexation and potential for delivery in vivo. In this regard, we have recently demonstrated that the polycation flexibility influences the complexation with single stranded RNA molecules, affecting the delivery capability of the resulting micelles. On the other hand, the effects of the catiomer flexibility on micelles loading double stranded siRNA remains unknown. Thus, herein, we studied the effects of the polycation backbone flexibility on siRNA-loaded polyion complex (PIC) micelles by using complementary block copolymers, i.e., the relatively flexible poly(ethylene glycol)-poly(glycidylbutylamine) (PEG-PGBA) and the more rigid PEG-poly(L-lysine) (PEG-PLL). By mixing these polymers with siRNA at different $\mathrm{N} / \mathrm{P}$ ratios, we found that PEG-PGBA effectively promoted self-assembly of PIC micelles at lower N/P ratios and lower siRNA concentrations than PEG-PLL. Computational studies of siRNA binding with polycations and PEG-polycations further supported the favorable binding process of flexible polycations with siRNA. The micelles based on PEG-PGBA were stable in physiological conditions and promoted effective intracellular delivery of siRNA for efficient gene knockdown. Our results indicate the importance of polycation flexibility for the assembly of PIC micelles with siRNA, and its potential for developing innovative carrier systems.
\end{abstract}

Keywords: small interfering RNAs (siRNAs); polyion complex (PIC); flexibility; molecular dynamics (MD) simulation 